\title{
Human amniotic membrane effect on colorectal anastomosis in dogs undergoing radiotherapy with/ without diverting stoma: An experimental preliminary study
}

\author{
S.V. Hosseini ${ }^{1}$, M. Dehghani ${ }^{1}$, H. Khazraei ${ }^{{ }^{*}}$, M.Y. Karami ${ }^{2}$, \\ S. Karbasi ${ }^{3}$; S.H. Banihashemi ${ }^{4}$ \\ ${ }^{1}$ Colorectal Research Center, Shiraz University of Medical Sciences, Shiraz, Iran \\ ${ }^{2}$ Student Research Committee, Shiraz University of Medical Sciences, Shiraz, Iran \\ ${ }^{3}$ Department of Radiotherapy and Oncology, Shiraz University of Medical Sciences, Shiraz, Iran \\ ${ }^{4}$ Department of surgery, Faculty of Medicine, Hormozgan University of Medical Sciences, Bandar Abbas, Iran
}

\section{- Short report}

\section{*Corresponding author:}

Dr. Hajar Khazraei,

Fax: +987136281453

E-mail:

hajarkhazraei@gmail.com

Revised: April 2016

Accepted: June 2016

Int. J. Radiat. Res., April 2017; 15(2): 213-218

DOI: 10.18869 /acadpub.ijrr.15.2.213

\begin{abstract}
Background: : Radiotherapy is an important factor which results in increase of anastomosis leakage. Diverting loop ileostomy has been usually performed after colorectal anastomosis with history of neo-adjuvant radiotherapy to decrease the chance of leakage. Considering this effect, we assessed the feasibility and outcome of human amniotic membrane in rectal anastomosis in dogs previously treated by high-dose radiotherapy. Materials and Methods: Twelve cross-breed male dogs with the age of 6-8 months and weighting 10-15 kg were randomly divided into four groups. Groups 1 and 2 received radiotherapy and 4 weeks later, single layer end to end anastomosis was performed for all the dogs; also, diverting loop ileostomy was placed in groups 1 and 3. Four weeks later, the anastomosis site was resected and sent for pathologic wound healing scoring. Data were analyzed by SPSS 16 software using Mann- Whitney test. Results: Two dogs (of group 1 and 2) died of peritonitis due to anastomosis leakage during the 2 nd post operation week. There was no statistically significant difference in wound healing between the case and control groups (P-Value: 0.01). Conclusion: HAM had a protective role in colorectal anastomosis after neo-adjuvant radiation in cases without loop diverting ileostomy. It can be concluded that HAM placement is a feasible technique instead of diverting loop ileostomy in cases with neoadjuvant radiotherapy and also in the benign colorectal diseases with high risk anastomoses.
\end{abstract}

Keywords: Human amniotic membrane, radiation therapy, anastomosis leakage, ileostomy.

\section{INTRODUCTION}

Colorectal anastomosis leakage is still one of the most atrocious complications $(2.4 \%$ to $69 \%)$ of colorectal procedure, which causes mortality (overall rates $7-12 \%$ ) and severe morbidity (rates 20-30\%) (1-3). Neoadjuvant radiotherapy has been applied in advanced rectal cancer to reach down staging and declining the rate of local recurrence. Preoperative radiotherapy may lead to deferred wound healing and following wound complication (4). Generally, changes in the fibroblast, vasculature, and growth factors function after radiotherapy initiate atypical wound-healing (5). Over expression of factors such as Tissue Growth Factor beta (TGF $\beta$ ), tumor necrosis factor- $\alpha$ (TNF- $\alpha$ ), vascular endothelial growth factor (VEGF), 
pro-inflammatory cytokines like interleukin-1 and interleukin- 8 and interferon- $\gamma($ IFN- $\gamma$ ), after irradiation, leads to uncontrolled matrix accumulation and fibrosis (6).

Amniotic membrane (AM), the inner layer of placenta, has important properties including antimicrobial, anti-inflammatory, antiscarring, antifibrosis, and low immunogenicity characteristics, and high potency of differentiation. Considering these features, the AM has already been used as a biologic dressing to promote wound healing process (4). Human Amniotic Membrane (HAM) was firstly applied in Johns Hopkins Hospital for treatment of 550 patients who had skin injuries. Since then, the HAM has been considered as a good treatment for tissue reconstruction, abdominal adhesiolysis, neurolysis, tenolysis and injuries of the vagina and dura matter (7).

Classic surgical approach to rectal cancer is neoadjuvant radiotherapy, segmental resection of the involved rectum with free margins, colorectal anastomosis, and diverting loop ileostomy except in Stage I (8).

Considering the side effects, complications and limitations of ileostomy include electrolytes imbalance, paraostomal hernia, and periostomal skin irritation, abnormal bowel habit mechanism, and psychosocial problems related to ostoma $(7,9,10)$. We can do colorectal surgery without using the diverting ileostomy and the rate of such complication will be reduced. In this study, we aimed to examine the effect of HAM on prevention of colorectal anastomosis leakage after neoadjuvant radiotherapy in dogs; this procedure could guide us for use of HAM in humans.

\section{MATERIALS AND METHODS}

In this prospective animal study, 12 cross-breed male dogs aged 6-8 months and weighing $10-15 \mathrm{~kg}$ were selected from the dogs in the animal laboratory of Shiraz University of Medical Sciences. All procedures were performed under aseptic conditions in accordance with the standards of laboratory bio-safety guidelines. This study was approved by Research and Ethics committee of Shiraz University of Medical Sciences.

The dogs $(\mathrm{N}=12)$ were randomly divided into four groups $(\mathrm{N}=3)$. Group 1 and 2 received external beam X-ray irradiation: Pelvic irradiation was performed at the radiation oncology department of Namazi hospital. External beam X-ray irradiation was carried out by a $6 \mathrm{MV}$ linear accelerator unit and under general anesthesia by intravenous Diazepam (10 $\mathrm{mg}$ ) and Sodium Thiopental $(0.5 \mathrm{mg})$ after endotracheal intubation. Then the dogs (groups 1 and 2) received a single fraction dose of $10 \mathrm{~Gy}$ through two parallel-opposed portals encompassing dog colorectum. The abdominal region containing approximately a $15 \mathrm{~cm}$ length of colorectum was exposed. We had ethical restrictions to include more than 8 dogs for external beam X-ray radiation. Groups 3 and 4 did not receive pre-operation radiotherapy.

After 4 weeks, all the dogs had anastomosis: animals were maintained on controlled ventilation by halothane and $100 \%$ oxygen. Ringer's lactate was administered throughout the operation at a rate of $8 \mathrm{~mL} / \mathrm{kg} / \mathrm{h}$ intravenously. Before induction, a single dose of antibiotic (Ceftriaxone, 1gr) was administered IV to the dogs. Then mid rectum $(15 \mathrm{~cm}$ above dentate line) sharp 360-degree perforation and single layer end to end anastomosis with vicryl 3.0 was performed. Animals did not receive mechanical or chemical bowel preparation.

Diverting loop ileostomy was placed for groups 1 and 3 and matured with vicryl 3.0. Then, it was fixed to the skin by a rod in the right lower quadrant of the dogs' abdomen. After irrigation and hemostasis, abdominal cavity was closed anatomically with vicryl 3.0 and the skin was closed in subcuticular manner with vicryl 3.0 .

In all dogs, a $2 \mathrm{~cm}$ width wrap of HAM was applied and fixed around the site of anastomosis with vicryl 3.0. Four weeks later, the anastomosis site was resected and again anastomosis was performed and the dogs were alive. Specimens were fixed in formaldehyde $10 \%$ solution (formalin). Samples were blindly labeled and sent to the department of pathology in Shiraz faculty of medicine for 
histopathological assessment. The HAM used in this study was provided by Shiraz Burn Research center, preserved in glutaraldehyde, and then frozen in $-20^{\circ} \mathrm{C}$.

After gross examination, histological sampling, tissue processing, slide preparation and $\mathrm{H}$ and $\mathrm{E}$ staining were performed. The slides were blindly studied by a pathologist. Histopathological findings were discussed for each sample on the basis of our modified scoring system. The scoring system was based on previous one which was suggested by Abramov et al. (11). The major modified item used in this system was histological evidence of epithelialization, collagenization and neovascularization.

\section{Statistical analysis}

SPSS for Windows version 16 statistical software (SPSS, Chicago, IL) was usedto perform the statistical analyses. Mann-Whitney $U$ test was performed, due to small sample size, to compare the groups and the normal distribution of data was examined by Kolmogorov-Smirnov test $(\mathrm{P}=0.03)$. Two-tailed $\mathrm{P}$-value less than 0.05 was considered statistically significant.

\section{RESULTS}

Two dogs (one of groups 1 and 2) died of peritonitis due to anastomosis leakage during the 2 nd post operation week. All others survived to the end of the study. After the second laparotomy, in gross evaluation of both groups, no patch disruption occurred, fistula, evidence of abscess formation, and pussy discharge or anastomosis site leakage was detected in the site of anastomosis.

Histological assessing of Group 1 and 2 demonstrated in table 1.

There was no statistically significant difference in wound healing between both groups $(\mathrm{P}>0.05)$, so wound healing process in ileostomy and non-ileostomy groups with neo-adjuvant radiotherapy had no statistically significant difference.

Microscopic evaluation of the harvested samples was performed by a pathologist. Modified scoring system for surgical wound healing (11) was used to determine the grade of healing in each sample.

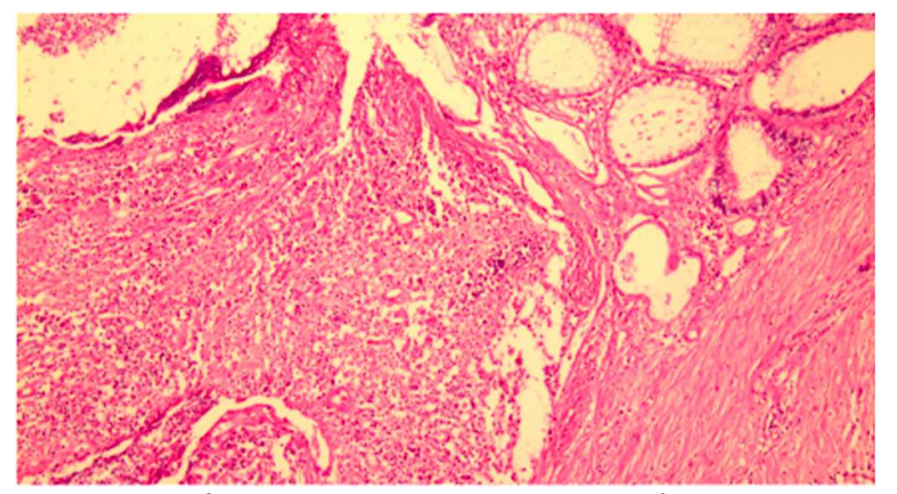

Figure 1. Mucosal ulceration with acute inflammation and granulation tissue formation. H\&E staining; magnification $\times 100$.

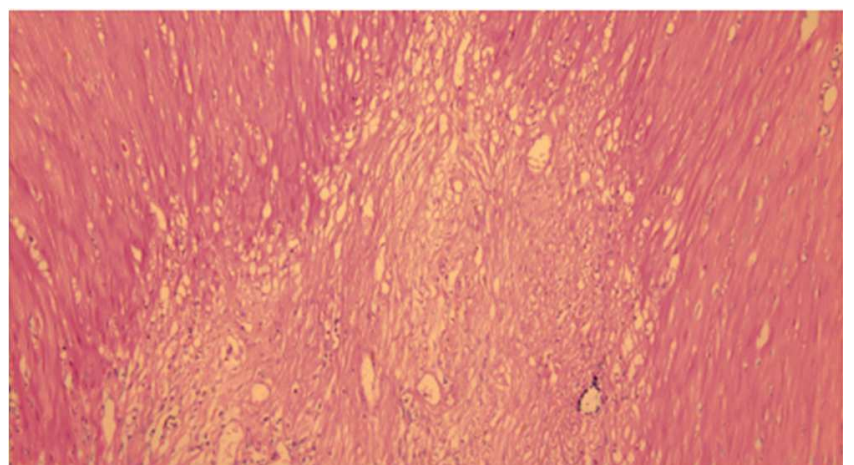

Figure 2. Mature fibrous tissue between muscle fibers suggestive of complete healing. H\&E staining; magnification $\times 100$. 
Table 1. Healing score according to collagenization, epithelialization, inflammation, neovascularization and necrosis of the samples.

\begin{tabular}{|c|c|c|c|c|}
\hline Groups & Colorectal Anastomosis & HAM & Radiotherapy & Ileostomy \\
\hline Group 1 & + & + & + & + \\
\hline Group 2 & + & + & + & - \\
\hline Group 3 & + & + & - & + \\
\hline Group 4 & + & + & - & - \\
\hline
\end{tabular}

Table 2. Histopathologic findings according Abramov wound healing scoring system in groups.

\begin{tabular}{|c|c|c|c|c|c|c|}
\hline Groups & $\begin{array}{c}\mathbf{1}(\text { Mean } \pm \text { SD) } \\
(\mathbf{n}=\mathbf{3})\end{array}$ & $\begin{array}{c}\mathbf{2}(\text { Mean } \pm \text { SD) } \\
(\mathbf{n}=\mathbf{3})\end{array}$ & $\begin{array}{c}\text { P-value between } \\
\text { groups 1,2 }\end{array}$ & $\begin{array}{c}\mathbf{3} \text { (Mean } \pm \text { SD) } \\
(\mathbf{n}=\mathbf{3})\end{array}$ & $\begin{array}{c}\mathbf{4}(\text { Mean } \pm \text { SD) } \\
(\mathbf{n}=\mathbf{3})\end{array}$ & $\begin{array}{c}\text { P-value between } \\
\text { group 3,4 }\end{array}$ \\
\hline Epithelialization & $0.66 \pm 0.57$ & $1.00 \pm 0.00$ & 0.31 & $1.00 \pm 0.00$ & $1.00 \pm 0.00$ & $>0.99$ \\
\hline Collagenization & $1.33 \pm 0.57$ & $2.66 \pm 0.57$ & 0.06 & $2.33 \pm 0.57$ & $2.00 \pm 0.00$ & 0.31 \\
\hline Inflammation & $3.00 \pm 0.00$ & $1.66 \pm 1.15$ & 0.11 & $2.33 \pm 1.15$ & $3.00 \pm 0.00$ & 0.31 \\
\hline Neovascularization & $1.00 \pm 0.00$ & $1.33 \pm 0.57$ & 0.31 & $1.66 \pm 0.57$ & $2.33 \pm 1.15$ & 0.36 \\
\hline Necrosis & $0.66 \pm 0.57$ & $0.33 \pm 0.57$ & 0.45 & $0.00 \pm 0.00$ & $0.00 \pm 0.00$ & $>0.99$ \\
\hline $\begin{array}{c}\text { Granulation } \\
\text { Tissue }\end{array}$ & $1.00 \pm 0.00$ & $2.33 \pm 1.15$ & 0.11 & $2.00 \pm 0.00$ & $1.66 \pm 0.57$ & 0.31 \\
\hline
\end{tabular}

Healing was scored according to collagenization, epithelialization, inflammation, neovascularization and necrosis of the samples. Pathologic evaluation revealed more significant epithelialization and neovascularization in group 2 (table 2). Overall, the healing score was higher in group $2(\mathrm{P}=0.31)$.

\section{DISCUSSION}

Anastomotic leakage is one of the most important complications that affecting $2-10 \%$ of patients undergoing colorectal surgery (9). Anastomotic dehiscence could be considered as a chief reason of extensive mortality (7\%-10\%) and morbidity (20\%-30\%) and unfortunate long term result $(12,13)$. Challenging wound healing caused by radiotherapy is commonly unnoticed during treatment of cancer. Chronic ulceration, infection, pain, psychological suffering and poor quality of life are clinical sequelea of deprived wound healing in patients undergoing radiotherapy (14).

Bio-compatibility, elasticity, easy availability and stability are some characteristics of HAM that cause growing propensity towards application of HAM as a biologic dressing to support wound healing specially in burn treatments (15). Our result can be discussed from two different perspectives. Firstly, the fact that our findings are generally in concordance and similar with the results of other studies which reported the application of HAM. In this regard, Barlas et al. mentioned the effectiveness of HAM as an intestinal patch for neomucosal growth in rabbits (16). Also, Najibpour et al.'s study on dogs indicated that applying HAM led to better histological outcomes and wound healing in colonic anastomosis compared to simple anastomosis (17). Roshanravan et al. applied HAM as a bio-prosthesis to repair recto-vaginal fistula in dogs; better surgical and histological outcomes were obtained (18).

In a study on 50 wistar rats, it was proved that the HAM, while contributing to reestablishment of duodenal wall structure, led to accelerated wound healing (19). HAM used as a temporary seal of damaged area in duodenal and it underwent degenerative changes in the matrix and in the epithelium after a week and eliminated. In our results, HAM eliminated and it could 
not be distinguish after a week and healing the lesions occurred.

Similarly, Uludag et al. reported lower dehiscence rate, leakage, and better wound healing after application of HAM in colonic anastomosis of rat (1). He introduced HAM patch in colon anastomosis in rats as treatment of dehiscence rate, anastomotic leakage, intra abdominal abscesses, intestinal obstruction and adhesion formation. Also, Kuriu et al.'s study showed that the intraperitoneal adhesion was reduced by HAM graft in rats (20).

Moreover, HAM has been used in reconstruction of the oral cavity, renovation of conjunctival defects, and prevention of postoperative adhesions $(21,22)$. Duodenal perforation repaired in Ghahramani et al.'s study (9) by HAM with better histological outcome.

Secondly, the application of HAM after radiation should be taken into consideration. Up on our knowledge, there were few studies assessed the efficacy of HAM after radiation in comparison to some other methods (6). The precise mechanism of HAM to reduce inflammation and increase wound healing process has not been completely clear yet; it seems that HAM works by suppressing inflammatory cytokines, expressing antiinflammatory proteins, tissue growth factor, multi-potent cells, and increasing angiogenesis and fibroblast activity $(1,23-25)$.

Finally, the application of HAM patch in repairing anastomosis leakage in dogs in our study clarified the advantages of HAM (as a biologically active treatment) in better surgical and histological outcomes comparing to simple repair.

\section{Limitations}

This trial was conducted on small size animals according to ethics committee restricted the induction of neoadjuvant radiotherapy for more than 8 dogs. Because of the similarity between human being and dogs (regarding GI tract), it is better to apply this method in human cases.

\section{CONCLUSION}

This experimental study was performed to determine the effect of HAM on wound healing in animal model undergoing neoadjuvant radiotherapy with and without protective loop ileostomy. Findings showed no statistically significant difference between groups with and without ileostomy about histologic and surgical outcome and leakage, so it is recommended that surgeons could use HAM in patients with neoadjuvant radiotherapy without an ileostomy creation.

Considering the inevitable negative effects of radiotherapy on wound healing, the use of HAM as a biologic dressing might be a feasible alternative to diverting loop ileostomy.

\section{ACKNOWLEDGEMENTS}

This study extracts from Dr. Dehghani thesis (no.8699). We would like to express our sincere gratitude to the Shiraz Uni-versity of Medical Sciences.

\section{Conflicts of interest: Declared none.}

\section{REFERENCES}

1. Uludag M, Citgez B, Ozkaya O, Yetkin G, Ozcan O, Polat N, et al. (2009) Effects of amniotic membrane on the healing of primary colonic anastomoses in the cecal ligation and puncture model of secondary peritonitis in rats. Int $\mathrm{J}$ Colorec Dis, 24(5): 559-67.

2. Corman ML, I.Allison S, P.Kuehne J (2005) Colon and Rectal Surgery. Colon and Rectal Surgery. New York: Lippincott Williams \& Wilkins, p. 177-277.

3. Kruschewski M, Rieger $H$, Pohlen $U$, Hotz HG, Buhr HJ (2007) Risk factors for clinical anastomotic leakage and postoperative mortality in elective surgery for rectal cancer. Int J Colorec Dis, 22(8): 919-27.

4. Niknejad H, Peirovi H, Jorjani M, Ahmadiani A, Ghanavi J, Seifalian AM (2008) Properties of the amniotic membrane for potential use in tissue engineering. European Cells \& Materials, 15: 88-99. 
5. Tibbs MK (1997) Wound healing following radiation therapy: a review. Radiotherapy and oncology. Journal of the European Society for Therapeutic Radiology and Oncology, 42(2):99-106.

6. Haubner F, Ohmann E, Pohl F, Strutz J, Gassner HG (2012) Wound healing after radiation therapy: review of the literature. Radiation Oncology, 7: 162.

7. Beck DE, Roberts PL, Saclarides TJ, Senagore AJ, Stamos MJ, Wexner SD (2011) The ASCRS textbook of Colon and Rectal Surgery. 2th ed., Springer Science and Business Media, New York.

8. Tsai BM, Finne CO, Nordenstam JF, Christoforidis D, Madoff RD, Mellgren A (2010) Transanal endoscopic microsurgery resection of rectal tumors: outcomes and recommendations. Dis Colon Rectum, 53(1): 16-23.

9. Telem DA, Chin EH, Nguyen SQ, Divino CM (2010) Risk factors for anastomotic leak following colorectal surgery: a case-control study. Archives of surgery (Chicago, III : 1960), 145(4): 371-6.

10. DKA FCB, R Billiar T, Dunn DL, G Hunter J, B Matthews J, Pollock RE (2010) Schwartz principles of surgery. 9th ed. McGraw-Hill, USA.

11. Abramov $Y$, Golden B, Sullivan M, Botros SM, Miller JJ, Alshahrour A, et al. (2007) Histologic characterization of vaginal vs. abdominal surgical wound healing in a rabbit model. Wound Repair and Regeneration, 15(1): 80-6.

12. McArdle CS, McMillan DC, Hole DJ (2005) Impact ofanastomotic leakage on long-term survival of patients undergoing curative resection for colorectal cancer. The British Journal of Surgery, 92(9): 1150-4.

13. Bell SW, Walker KG, Rickard MJ, Sinclair G, Dent OF, Chapuis PH, et al. (2003) Anastomotic leakage after curative anterior resection results in a higher prevalence of local recurrence. The British Journal of Surgery, 90(10): 1261-6.

14. Dormand EL, Banwell PE, Goodacre TE (2005) Radiotherapy and wound healing. International Wound Journal, 2(2): 112-27.

15. Kesting MR, Wolff KD, Hohlweg-Majert B, Steinstraesser L (2008) The role of allogenic amniotic membrane in burn treatment. Journal of Burn Care \& Research, 29(6): 90716.

16. Barlas M, Gokcora H, Erekul S, Dindar H, Yucesan S (1992) Human amniotic membrane as an intestinal patch for neomucosal growth in the rabbit model. Journal of Pediatric Surgery, 27(5): 597-601.

17. Najibpour N, Jahantab MB, Hosseinzadeh M, Roshanravan R, Moslemi S, Rahimikazerooni S, et al. (2013) The Effects of Human Amniotic Membrane on Healing of Colonic Anastomosis in Dogs. Annals of Colorectal Research, 1(3): 97-100.

18. Roshanravan R, Ghahramani L, Hosseinzadeh M, Mohammadipour M, Moslemi S, Rezaianzadeh A, et al. (2014) A new method to repair recto-vaginal fistula: Use of human amniotic membrane in an animal model. Advanced Biomedical Research, 3: 114.

19. Schimidt LR, Cardoso EJ, Schimidt RR, Back LA, Schiazawa $M B$, d'Acampora AJ, et al. (2010) The use of amniotic membrane in the repair of duodenal wounds in Wistar rats. Acta cirurgica brasileira / Sociedade Brasileira para Desenvolvimento Pesquisa em Cirurgia, 25(1): 18-23.

20. Kuriu $Y$, Yamagishi H, Otsuji E, Nakashima S, Miyagawa K, Yoshikawa T, et al. (2009) Regeneration of peritoneum using amniotic membrane to prevent postoperative adhesions. Hepato-gastroenterology, 56(93): 1064-8.

21. von Versen-Hoynck F, Hesselbarth U, Moller DE (2004) Application of sterilised human amnion for reconstruction of the ocular surface. Cell and Tissue Banking, 5(1): 57-65.

22. Szabo A, Haj M, Waxsman I, Eitan A (2000) Evaluation of seprafilm and amniotic membrane as adhesion prophylaxis in mesh repair of abdominal wall hernia in rats. European Surgical Research, 32(2): 125-8.

23. Uludag M, Ozdilli K, Citgez B, Yetkin G, Ipcioglu OM, Ozcan $O$, et al. (2010) Covering the colon anastomoses with amniotic membrane prevents the negative effects of early intraperitoneal 5-FU administration on anastomotic healing. International Journal of Colorectal Disease, 25(2): 223 -32 .

24. Uludag M, Citgez B, Ozkaya O, Yetkin G, Ozcan O, Polat N, et al. (2009) Effects of amniotic membrane on the healing of normal and high-risk colonic anastomoses in rats. International Journal of Colorectal Disease, 24(7):809-17.

25. Kruse FE and Meller D (2001) Amniotic membrane transplantation for reconstruction of the ocular surface. Der Ophthalmologe: Zeitschrift der Deutschen Ophthalmologischen Gesellschaft, 98(9): 801-10. 